\title{
Constant thrust programming for a multistage rocket flown in vacuum with a given initial/final thrust-to-weight ratio in an arbitrary stage
}

\author{
S N MAITRA \\ Department of Mathematics, National Defence Academy, P.O. NDA Khadakwasla, \\ Pune 411023, India
}

MS received 13 July 1984; revised 30 November 1984

\begin{abstract}
The burn time and burnout velocity of a multistage rocket flown vertically in vacuum with constant thrust tangential to the flight path and a prescribed initial/final thrustto-weight ratio in an arbitrary stage have been determined.

The present paper also deals with optimal staging under given conditions of flight.
\end{abstract}

Keywords. Constant thrust programming; multistage rocket; thrust-to-weight ratio; burntime, burnout velocity.

\section{Introduction}

Miele (1962) derived the formulae for the burn time and burnout velocity of a multistage rocket flown vertically with the thrust tangential to the flight path where the rocket is designed in such a way that (a) the thrust of each stage is constant (whether the initial/final thrust-to-weight ratio of each stage is constant or varies from stage to stage), and the initial thrust-to-weight ratio of each stage is prescribed, (b) the thrust of each stage is constant and the final thrust-to-weight ratio of each stage is prescribed. Both Barrere (1960) and Ruppe (1966) studied optimisation of partial mass ratios ledding to the maximum over-all payload ratio/burnout velocity.

The present analysis deals with the multistage performance in vacuum (a) with a constant thrust throughout in the burn phase and a prescribed initial thrust-to-weight ratio in the $r$ th stage; (b) with a constant thrust throughout the burn phase and a prescribed final thrust-to-weight ratio in the $r$ th stage, including the optimisation of partial payload ratios for attaining the maximum over-all burnout velocity or payload ratio in both the cases.

\section{Overall burn time and burnout velocity with a prescribed initial thrust-to-weight ratio in the $r$ th stage}

If $\beta_{k}, V_{E k}, m_{i k}, \tau_{i k}, g$ and $n$ be the propellant mass flow, the equivalent exhaust velocity, the initial mass, the initial thrust-to-weight ratio in the $k$ th stage, the acceleration due to gravity and the number of stages, the constant thrust $T$ for the multistage performance can be expressed as

$$
\begin{aligned}
T / g & =m_{i 1} \tau_{i 1}=m_{i 2} \tau_{i 2}=\cdots=m_{i k} \tau_{i k}=\cdots=m_{i r} \tau_{i r}=\ldots=m_{i n} \tau_{i n} \\
& =\beta_{1} V_{E 1}=\beta_{2} V_{E 2}=\cdots=\beta_{k} V_{E k}=\cdots=\beta_{r} V_{E r}=\ldots=\beta_{n} V_{E n},
\end{aligned}
$$


denoted by

$$
\pi_{k}=m_{i(k+1)} / m_{i k}, \zeta_{k}=m_{p k} / m_{i k}, \varepsilon_{k}=m_{s k} /\left(m_{s k}+m_{p k}\right),
$$

the payload ratio, the propellant mass ratio, and the structural factor respectively in the $k$ th stage where $m_{p k}$ and $m_{s k}$ are the propellant mass and the structural mass respectively in that stage. If $m_{f k}$ be the final mass in the $k$ th stage,

$$
m_{f k}=m_{i k}-m_{p k} \text {. }
$$

As a consequence of (1) and (2), the following relationships will hold good:

$$
\begin{aligned}
\tau_{i k} & =\tau_{i(k+1)} m_{i(k+1)} / m_{i k} \\
& =\tau_{i(k+1)} \pi_{k} \\
& =\tau_{i(k+2)} \pi_{k+1} \pi_{k} \\
& =\tau_{i r} \pi_{k} \pi_{k+1} \ldots \pi_{r-1},
\end{aligned}
$$

where $\quad r>k$, and $\tau_{i k}=\tau_{i r}$ when $r=k$.

Further,

where $r<k$.

$$
\begin{aligned}
\tau_{i k} & =\tau_{i(k-1)} m_{i(k-1)} / m_{i k} \\
& =\tau_{i(k-1)} / \pi_{k-1} \\
& =\tau_{i(k-2)} / \pi_{k-1} \pi_{k-2} \\
& =\tau_{i r} /\left(\pi_{k-1} \pi_{k-2} \ldots \pi_{r}\right)
\end{aligned}
$$

The overall burn time is given by

$$
t_{*}=\sum_{k=1}^{n} m_{p k} / \beta_{k}
$$

Because of (1) this is

$$
t_{*}=(1 / g) \sum_{k=1}^{n} V_{E k} m_{P k} / \tau_{i k} m_{i k}
$$

and because of (2)-(5)

$$
\begin{aligned}
t_{*}= & \frac{1}{g} \sum_{k=1}^{r-1} \frac{V_{E k}\left(1-\varepsilon_{k}\right)\left(1-\pi_{k}\right)}{\tau_{i r} \pi_{k} \pi_{k+1} \ldots \pi_{r-1}}+\frac{V_{E r}\left(1-\varepsilon_{r}\right)\left(1-\pi_{r}\right)}{g \tau_{i r}} \\
& +\frac{1}{g} \sum_{k=r+1}^{n} \frac{V_{E k}\left(1-\varepsilon_{k}\right)\left(1-\pi_{k}\right) \pi_{k-1} \pi_{k}-2 \ldots \pi_{r}}{\tau_{i r}} .
\end{aligned}
$$

If the characteristic parameters of each stage are identical such that

$$
V_{E k}=V_{E}, \pi_{k}=\pi, \varepsilon_{k}=\varepsilon,
$$

(6) simplifies to the form,

$$
t_{*}=V_{E}(1-\varepsilon)\left(1-\pi_{0}\right) /\left(g \tau_{i r} \pi_{0^{n}}^{\frac{e^{-1}}{1}}\right),
$$

where the overall payload ratio is given by

$$
\pi_{0}=\pi^{n}
$$


The dimensionless burn time is

$$
\theta=(1-\varepsilon)\left(1-\pi_{0}\right) /\left(\tau_{i r} \pi_{0}^{\frac{c-1}{n}}\right)
$$

Hence, with a prescribed initial thrust-to-weight ratio in the first stage or with a prescribed initial thrust-to-weight ratio in the last stage, the burn time becomes

or

$$
\begin{aligned}
& \theta_{1}=(1-\varepsilon)\left(1-\pi_{0}\right) / \tau_{i 1}, \\
& \theta_{n}=(1-\varepsilon)\left(1-\pi_{0}\right) /\left(\tau_{i n} \pi_{0}^{\frac{n-1}{n}}\right) .
\end{aligned}
$$

In (12),

$$
\lim _{n \rightarrow \infty} \theta_{n}=(1-\varepsilon)\left(1-\pi_{0}\right) /\left(\tau_{i n} \pi_{0}\right)
$$

i.e., as the number of stages increases, the burn time increases for given values of the structural factor, the overall payload ratio and the initial thrust-to-weight ratio in the last stage, and acquires a limiting value as $n \rightarrow \infty$.

The burnout velocity corresponding to the gravity-included case, where the rocket starts from rest and the final velocity of each stage is equal to the inital velocity of the next stage, is obtained as

$$
V_{*}=\sum_{k=1}^{n} V_{E k} \log \left(m_{i k} / m_{f k}\right)-g t_{*},
$$

using (2)-(6),

$$
\begin{aligned}
V_{*}= & \sum_{k=1}^{n} V_{E k} \log 1 /\left[\varepsilon_{k}+\left(1-\varepsilon_{k}\right) \pi_{k}\right]-\left[V_{E r}\left(1-\varepsilon_{r}\right)\left(1-\pi_{r}\right) / \tau_{i r}\right] \\
& -\sum_{k=1}^{r-1}\left[V_{E k}\left(1-\varepsilon_{k}\right)\left(1-\pi_{k}\right)\right] /\left[\tau_{i r} \pi_{k} \pi_{k+1} \ldots \pi_{r-1}\right] \\
& -\sum_{k=r+1}^{n}\left[V_{E k}\left(1-\varepsilon_{k}\right)\left(1-\pi_{k}\right) \pi_{k-1} \pi_{k-2} \ldots \pi_{r}\right] / \tau_{i r}
\end{aligned}
$$

By virtue of (7)-(9)

$$
V_{*}=V_{E} n \log 1 /\left[\varepsilon+(1-\varepsilon) \pi_{b}^{j}\right]-\left[V_{E}(1-\varepsilon)\left(1-\pi_{0}\right) /\left(\tau_{i r} \pi_{0}^{\frac{r-1}{x}}\right)\right]
$$

and the dimensionless burnout velocity is

$$
u=n \log 1 /\left[\varepsilon+(1-\varepsilon) \pi_{0}^{\frac{1}{i}}\right]-(1-\varepsilon)\left(1-\pi_{0}\right) /\left(\tau_{i r} \pi_{0}^{r^{n-1}}\right) .
$$

If $u_{0}$ denotes the non-dimensional velocity in the gravity-free case, (16) yields

$$
u=u_{0}\left(\pi_{0}, n, \varepsilon\right)-\theta\left(\pi_{0}, n, \varepsilon, \tau_{i r}\right),
$$

From (16) it is clear that (a) for each given velocity, the payload ratio increases with the number of stages and (b) for each given payload ratio, the burnout velocity increases with the number of stages.

\section{Overall burn time and burnout velocity with a prescribed final thrust-to-weight ratio in the rth stage}

Besides (1), the following equation gives the constant thrust with the prescribed final 
thrust-to-weight ratio in the $r$ th stage:

$$
T / g=m_{f 1} \tau_{f 1}=m_{f 2} \tau_{f 2}=\ldots=m_{f k} \tau_{f k}=\ldots=m_{f r} \tau_{f r}=\ldots m_{f n} \tau_{f n}
$$

where $m_{f r}$ and $\tau_{f r}$ are the final mass and the final thrust-to-weight ratio in the rth stage.

Combining (18) with (2), one can find

$$
\begin{aligned}
\tau_{f k} & =\tau_{f(k+1)} m_{f(k+1)} / m_{f k} \\
& =\tau_{f(k+1)}\left\{\left[\varepsilon_{k+1}+\left(1-\varepsilon_{k+1}\right) \pi_{k+1}\right] /\left[\varepsilon_{k}+\left(1-\varepsilon_{k}\right) \pi_{k}\right]\right\} \pi_{k} \\
& =\tau_{f(k+2)}\left\{\left[\varepsilon_{k+2}+\left(1-\varepsilon_{k+2}\right) \pi_{k+2}\right] /\left[\varepsilon_{k}+\left(1-\varepsilon_{k}\right) \pi_{k}\right]\right\} \pi_{k} \pi_{k+1} \\
& =\tau_{f r}\left\{\left[\varepsilon_{r}+\left(1-\varepsilon_{r}\right) \pi_{r}\right] /\left[\varepsilon_{k}+\left(1-\varepsilon_{k}\right) \pi_{k}\right]\right\} \pi_{k} \pi_{k+1} \pi_{k+2} \ldots \pi_{r-1},
\end{aligned}
$$

where $r>k \tau_{f k}=\tau_{f r}$ when $r=k$.

But if $r<k$,

$$
\begin{aligned}
\tau_{f k} & =\tau_{f(k-1)} m_{f(k-1)} / m_{f k} \\
& =\tau_{f(k-1)}\left[\varepsilon_{k-1}+\left(1-\varepsilon_{k-1}\right) \pi_{k-1}\right] /\left[\varepsilon_{k}+\left(1-\varepsilon_{k}\right) \pi_{k}\right] \pi_{k-1} \\
& =\tau_{f(k-2)}\left[\varepsilon_{k-2}+\left(1-\varepsilon_{k-2}\right) \pi_{k-2}\right] /\left[\varepsilon_{k}+\left(1-\varepsilon_{k}\right) \pi_{k}\right] \pi_{k-2} \pi_{k-1} \\
& =\tau_{f r}\left[\varepsilon_{r}+\left(1-\varepsilon_{r}\right) \pi_{r}\right] /\left\{\left[\varepsilon_{k}+\left(1-\varepsilon_{k}\right) \pi_{k}\right] \pi_{k-1} \pi_{k-2} \ldots \pi_{r}\right\} .
\end{aligned}
$$

Hence, in this section the overall burn period is

$$
t_{*}^{\prime}=\sum_{k=1}^{n}\left(V_{E k} m_{p k}\right) /\left(\tau_{f k} m_{f k}\right)
$$

which, by virtue of (1), (2), (18)- (20) becomes

$$
\begin{aligned}
t_{*}^{\prime}= & \frac{1}{g} \sum_{k=1}^{r-1} \frac{\left[V_{E k}\left(1-\varepsilon_{k}\right)\left(1-\pi_{k}\right)\right]}{\tau_{f r}\left[\varepsilon_{r}+\left(1-\varepsilon_{r}\right) \pi_{r}\right] \pi_{k} \pi_{k+1} \ldots \pi_{r-1}} \\
& +\frac{V_{E r}\left(1-\varepsilon_{r}\right)\left(1-\pi_{r}\right)}{g \tau_{f r}\left\{\varepsilon_{r}+\left(1-\varepsilon_{r}\right) \pi_{r}\right\}} \\
& +\frac{1}{g} \sum_{k=r+1}^{n} \frac{V_{E k}\left(1-\varepsilon_{k}\right)\left(1-\pi_{k}\right) \pi_{k-1} \pi_{k-2} \ldots \pi_{r}}{\tau_{f r}\left\{\varepsilon_{r}+\left(1-\varepsilon_{r}\right) \pi_{r}\right\}} .
\end{aligned}
$$

Owing to the conditions (7) and (9),

$$
t_{*}^{\prime}=\left[V_{E}(1-\varepsilon)\left(1-\pi_{0}\right)\right] /\left\{g \tau_{\rho r}\left[\varepsilon+(1-\varepsilon) \pi_{0}^{\frac{1}{2}}\right] \pi_{0}^{\frac{\tau_{0}^{*}}{*}}\right\}
$$

and the dimensionless burn period is

$$
\theta^{\prime}=\left[(1-\varepsilon)\left(1-\pi_{0}\right)\right] /\left\{\tau_{f r}\left[\varepsilon+(1-\varepsilon) \pi_{0}^{\frac{1}{b}}\right] \pi_{0}^{\frac{L^{-1}}{n}}\right\}
$$

The over-all burn time with the prescribed final thrust-to-weight ratio in the first stage and that with the prescribed final thrust-to-weight ratio in the last stage are respectively given by

$$
\theta_{1}^{\prime}=\left[(1-\varepsilon)\left(1-\pi_{0}\right)\right] /\left\{\tau_{f 1}\left[\varepsilon+(1-\varepsilon) \pi_{0}^{\frac{1}{2}}\right]\right\}
$$


and

$$
\theta_{n}^{\prime}=\left[(1-\varepsilon)\left(1-\pi_{0}\right) /\left\{\tau_{f n}\left[\varepsilon+(1-\varepsilon) \pi_{0}^{\frac{1}{b}}\right] \pi_{0}^{\frac{n-1}{n}}\right\}\right.
$$

As the number of stages increases, the burn time $\theta_{n}^{\prime}$ increases and has the limiting value:

$$
\operatorname{Lim}_{n \rightarrow \infty} \theta_{n}^{\prime}=(1-\varepsilon)\left(1-\pi_{0}\right) /\left(\tau_{f n} \pi_{0}\right)
$$

The burnout velocity can be given [as a consequence of (2), (3), (21)] by

$$
\begin{aligned}
V_{*}^{\prime}= & \sum_{k=1}^{n} V_{E k} \log \frac{1}{\varepsilon_{k}+\left(1-\varepsilon_{k}\right) \pi_{k}}-\frac{V_{E r}\left(1-\varepsilon_{r}\right)\left(1-\pi_{r}\right)}{\tau_{f r}\left\{\varepsilon_{r}+\left(1-\varepsilon_{r}\right) \pi_{r}\right\}} \\
& -\sum_{k=1}^{r-1} \frac{V_{E k}\left(1-\varepsilon_{k}\right)\left(1-\pi_{k}\right)}{\tau_{f r}\left\{\varepsilon_{r}+\left(1-\varepsilon_{r}\right) \pi_{r}\right\} \pi_{k} \pi_{k-1} \ldots \pi_{r-1}} \\
& -\sum_{k=r+1}^{n} \frac{V_{E k}\left(1-\varepsilon_{k}\right)\left(1-\pi_{k}\right) \pi_{k-1} \pi_{k-2} \ldots \pi_{r}}{\tau_{f r}\left\{\varepsilon_{r}+\left(1-\varepsilon_{r}\right) \pi_{r}\right\}}
\end{aligned}
$$

Using the conditions (7) and (9),

$$
V_{*}^{\prime}=n V_{E} \log \frac{1}{\varepsilon+(1-\varepsilon) \pi_{0}^{\frac{1}{n}}}-\frac{V_{E}\left(1-\pi_{0}\right)(1-\varepsilon)}{\tau_{f r} \pi_{0}^{\frac{r-1}{n}}\left\{\varepsilon+(1-\varepsilon) \pi_{0}^{\frac{1}{x}}\right\}}
$$

The dimensionless burnout velocity becomes

$$
u=n \log \frac{1}{\varepsilon+(1-\varepsilon) \pi_{0}^{\frac{1}{2}}}-\frac{(1-\varepsilon)\left(1-\pi_{0}\right)}{\tau_{f r} \pi_{0}^{\frac{\tau}{n}}\left\{\varepsilon+(1-\varepsilon) \pi_{0}^{\frac{1}{n}}\right\}}
$$

which has the form

$$
u=u_{0}\left(\pi_{0}, n, \varepsilon\right)-\theta\left(\pi_{0}, n, \varepsilon, \tau_{f r}\right)
$$

where $u_{0}$ is the burnout velocity in the gravity-free case. From (28), the same type of conclusion can be reached as from (16).

The relation between the initial and final thrust-to-weight ratios in the rth stage can be written as

$$
\tau_{i r} / \tau_{f r}=\varepsilon+(1-\varepsilon) \pi_{0}^{\frac{1}{k}}
$$

so that the burnout velocity can be rewritten as

$$
u=n \log \left[\tau_{f r} / \tau_{i r}\right]-\left[\left(1-\pi_{0}\right)\left(\tau_{f r}-\tau_{i r}\right) / \tau_{i r} \tau_{f r}\left(\pi_{0^{n}}^{\frac{r-1}{n}}-\pi_{0}^{i}\right)\right] .
$$

If $\tau_{i r}=1$ is taken, the following parametric equations can be obtained:

$$
\begin{aligned}
& u=n \log \tau_{f r}+\left[\left(1-\pi_{0}\right)\left(1-\tau_{f r}\right) / \tau_{f r}\left(\pi_{0^{k}}^{\frac{r-1}{k}}-\pi_{0}^{k}\right)\right], \\
& \pi_{0}=\left[\left(1-\varepsilon \tau_{f r}\right) /(1-\varepsilon) \tau_{f r}\right]^{n},
\end{aligned}
$$

which are valid subject to the restriction

$$
\tau_{f r}<1 / \varepsilon
$$

Elimination of the parameter $n$ from (33) leads to

$$
\begin{aligned}
u= & \left(\log \pi_{0}\right)\left(\log \tau_{f r}\right) / \log \left\{(1-\varepsilon) \tau_{f r} /\left(1-\varepsilon \tau_{f r}\right)\right\} \\
& -\left(1-\pi_{0}\right)\left\{(1-\varepsilon) \tau_{f r}\right\} / \tau_{f r}\left(1-\varepsilon \tau_{f r}\right)^{r-1}
\end{aligned}
$$


By means of (4), (5), (7), (19), (20) and (31)

$$
\tau_{i 1} / \tau_{f r}=\left\{\varepsilon+(1-\varepsilon) \pi_{0}^{\frac{1}{5}}\right\} \pi_{0}^{\frac{1-1}{\pi}} \text {. }
$$

Eliminating the structural factor $\varepsilon$ from (29) and (37) the burnout velocity can also be expressed as

$$
u=n \log \frac{\tau_{f r}}{\tau_{i 1}}+(r-1) \log \pi_{0}-\left[\left(\pi_{0}^{\frac{r-1}{n}} \tau_{f r}-\tau_{i 1}\right)\left(1-\pi_{0}\right) / \tau_{i 1} \tau_{f r}\left(\pi_{0}^{c-1}-\pi_{0}^{\frac{1}{*}}\right)\right]
$$

In the light of the foregoing analysis, the following observations can be made:

In view of the assumptions that $\varepsilon_{k}=\varepsilon, \pi_{k}=\pi, V_{E k}=V_{E}$ and $\tau_{i k}=\tau_{i}, \tau_{f k}=\tau_{f}$ in Miele's analysis, one can get

$$
\beta_{k+1} / \beta_{k}=m_{i(k+1)} / m_{i k}=\pi<1,
$$

i.e., the propellant mass flow decreases with the number of stages in the thrust programme associated with a prescribed initial thrust-to-weight ratio in each stage.

Similarly for the thrust programme associated with a prescribed final thrust-toweight ratio in each stage,

$$
\beta_{k+1} / \beta_{k}=m_{f(k+1)} / m_{f k}=\pi<1 .
$$

Hence, in this case also the propellant mass flow decreases with the number of stages.

In the present analysis, however, the thrust being constant throughout the powered flight, the propellant mass flow remains constant for constant equivalent exhaust velocity.

\section{Optimisation of partial payload ratios for maximum overall burnout velocity}

In the following sections the problem of optimisation of partial payload ratios is investigated while the multistage rocket is flown in vacuum with a constant thrust throughout the burn period with a given initial/final thrust-to-weight ratio in an arbitrary stage.

Case 1: Maintaining the same equivalent exhaust velocity $V_{E}$ in all stages, $\left(u=V / V_{E}\right)$. Equation (14) in the nondimensional form gives:

(i) For $j \leqslant r-1$,

$$
\begin{aligned}
\frac{\partial u}{\partial \pi_{j}}= & \frac{-\left(1-\varepsilon_{j}\right)}{\varepsilon_{j}+\left(1-\varepsilon_{j}\right) \pi_{j}}+\frac{1}{\tau_{i r}} \sum_{i=1}^{j-1} \frac{\left(1-\varepsilon_{i}\right)\left(1-\pi_{i}\right)}{\pi_{i} \pi_{i+1} \ldots \pi_{j}^{2} \ldots \pi_{r-1}} \\
& +\frac{\left(1-\varepsilon_{j}\right)}{\tau_{i r} \pi_{j}^{2} \pi_{j+1} \ldots \pi_{r-1}}
\end{aligned}
$$

(ii) For $j=r$,

$$
\frac{\partial u}{\partial \pi_{r}}=\frac{-\left(1-\varepsilon_{r}\right)}{\varepsilon_{r}+\left(1-\varepsilon_{r}\right) \pi_{r}}+\frac{1-\varepsilon_{r}}{\tau_{i r}}-\sum_{k=r+1}^{n} \frac{\left(1-\varepsilon_{k}\right)\left(1-\pi_{k}\right) \pi_{k-1} \pi_{k-2} \ldots \pi_{r}}{\tau_{i r} \pi_{r}} .
$$


(iii) For $r+1 \leqslant j \leqslant n-1$,

$$
\begin{aligned}
\frac{\partial u}{\partial \pi_{j}}= & -\left(1-\varepsilon_{j}\right) /\left\{\varepsilon_{j}+\left(1-\varepsilon_{j}\right) \pi_{j}\right\}-\sum_{i=j+1}^{n} \frac{\left(1-\varepsilon_{i}\right)\left(1-\pi_{i}\right) \pi_{i-1} \pi_{i-2} \ldots \pi_{r}}{\tau_{i r} \pi_{j}} \\
& +\frac{\left(1-\varepsilon_{j}\right) \pi_{j-1} \pi_{j-2} \ldots \pi_{r}}{\tau_{i r}}
\end{aligned}
$$

(iv) For $j=n$,

$$
\frac{\partial u}{\partial \pi_{n}}=\frac{-\left(1-\varepsilon_{n}\right)}{\varepsilon_{n}+\left(1-\varepsilon_{n}\right) \pi_{n}}+\frac{\left(1-\varepsilon_{n}\right) \pi_{r} \pi_{r+1} \ldots \pi_{n-1}}{\tau_{i r}} .
$$

Now, $\frac{\partial u}{\partial \pi_{j}}<0$ for all $\tau_{i r}>1, \varepsilon_{j}<1$ and $\pi_{j}<1$, where $j=r, r+1, \ldots n$.

By virtue of the foregoing relationships it can be established that for given values of $\varepsilon_{k}(k=1,2, \ldots n)$ there exists a set of $(r-1)$ partial payload ratios (preceding the $r$ th stage) which maximizes the overall burnout velocity with a prescribed initial thrust-toweight ratio in the $r$ th stage and satisfies the following equation:

$$
\frac{1-\varepsilon_{j}}{\varepsilon_{j}+\left(1-\varepsilon_{j}\right) \pi_{j}}-\frac{1}{\tau_{i r}} \sum_{i=1}^{j-1} \frac{\left(1-\varepsilon_{i}\right)\left(1-\pi_{i}\right)}{\pi_{i} \pi_{i+1} \ldots \pi_{j}^{2} \ldots \pi_{r-1}}-\frac{1-\varepsilon_{j}}{\tau_{i r} \pi_{j}^{2} \pi_{j+1} \ldots \pi_{r-1}}=0
$$

where $j=1,2,3, \ldots(r-1)$.

Case 2. Similarly, (27) in the nondimensional form gives the two following optimisation equations:

(i) For $j \leqslant r-1$,

$$
\begin{aligned}
\frac{\partial u}{\partial \pi_{j}}= & \frac{-\left(1-\varepsilon_{j}\right)}{\varepsilon_{j}+\left(1-\varepsilon_{j}\right) \pi_{j}}-\sum_{i=1}^{j-1} \frac{\left(1-\varepsilon_{i}\right)\left(1-\pi_{i}\right)}{\tau_{f r}\left\{\varepsilon_{r}+\left(1-\varepsilon_{r}\right) \pi_{r}\right\} \pi_{i} \pi_{i+1} \ldots \pi_{j}^{2} \ldots \pi_{r-1}} \\
& +\frac{1-\varepsilon_{j}}{\tau_{f r}\left\{\varepsilon_{r}+\left(1-\varepsilon_{r}\right) \pi_{r}\right\} \pi_{j}^{2}} \frac{\pi_{j+1} \ldots \pi_{r-1}}{\pi_{j+1}}=0 .
\end{aligned}
$$

(ii) For $j=r$,

$$
\begin{aligned}
\frac{\partial u}{\partial \pi_{r}}= & \frac{-\left(1-\varepsilon_{r}\right)}{\varepsilon_{r}+\left(1-\varepsilon_{r}\right) \pi_{r}}+\frac{1-\varepsilon_{r}}{\tau_{f r}\left\{\varepsilon_{r}+\left(1-\varepsilon_{r}\right) \pi_{r}\right\}^{2}} \\
& -\sum_{k=r+1}^{n} \frac{\varepsilon_{r}\left(1-\varepsilon_{k}\right)\left(1-\pi_{k}\right) \pi_{k-1} \pi_{k-2} \ldots \pi_{r}}{\tau_{f r}\left\{\varepsilon_{r}+\left(1-\varepsilon_{r}\right) \pi_{r}\right\}^{2} \pi_{r}}=0 .
\end{aligned}
$$

(iii) For $r+1 \leqslant j \leqslant n-1$,

$$
\begin{aligned}
\frac{\partial u}{\partial \pi_{j}}= & \frac{-\left(1-\varepsilon_{j}\right)}{\varepsilon_{j}+\left(1-\varepsilon_{j}\right) \pi_{j}}-\sum_{i=j+1}^{n} \frac{\left(1-\varepsilon_{i}\right)\left(1-\pi_{i}\right) \pi_{i-1} \pi_{i-2} \ldots \pi_{r}}{\tau_{f r}\left\{\varepsilon_{r}+\left(1-\varepsilon_{r}\right) \pi_{r}\right\} \pi_{j}} \\
& +\frac{\left(1-\varepsilon_{j}\right) \pi_{j-1} \pi_{j-2} \ldots \pi_{r}}{\tau_{f r}\left\{\varepsilon_{r}+\left(1-\varepsilon_{r}\right) \pi_{r}\right\}}<0 .
\end{aligned}
$$


(iv) For $j=n$,

$$
\begin{array}{r}
\frac{\partial u}{\partial \pi_{n}}=\frac{-\left(1-\varepsilon_{n}\right)}{\varepsilon_{n}+\left(1-\varepsilon_{n}\right) \pi_{n}}+\frac{\left(1-\varepsilon_{n}\right) \pi_{n-1} \ldots \pi_{r}}{\tau_{f r}\left\{\varepsilon_{r}+\left(1-\varepsilon_{r}\right) \pi_{r}\right\}}<0 . \\
\left(\because \tau_{i r}=\tau_{f r}\left\{\varepsilon_{r}+\left(1-\varepsilon_{r}\right) \pi_{r}\right\}>1 \text { for } r=1,2,3, \ldots n .\right)
\end{array}
$$

Thus, for given values of all partial structural factors and partial payload ratios of the last $(n-r)$ stages, the partial payload ratios of the first $r$ stages, which maximize the overall burnout velocity with a prescribed final thrust-to-weight ratio in the $r$ th stage, satisfy the foregoing equations (42a) and (42b).

If all partial structural factors are equal to $\varepsilon,(14)$ yields:

For $j \leqslant r-1$,

$$
\frac{\partial u}{\partial \pi_{j}}=-\frac{1}{\varepsilon+(1-\varepsilon) \pi_{j}}+\frac{1}{\tau_{i r} \pi_{1} \pi_{2} \pi_{3} \ldots \pi_{j-1} \pi_{j}^{2} \pi_{j+1} \ldots \pi_{r-1}}=0
$$

where $j=1,2,3 \ldots(r-1)$.

The above $(r-1)$ equations give $\pi_{j}=\pi$ for $j=1,2,3, \ldots(r-1)$,

i.e., payload ratios of the first $(r-1)$ stages are equal and satisfy the following equation in the first case:

$$
\varepsilon+(1-\varepsilon) \pi=\tau_{i r} \pi^{\prime}
$$

which ensures that for all $\tau_{i 1}=\tau_{i r} \pi^{r-1}>1, \pi<1$.

Obviously, if the initial thrust-to-weight ratio be prescribed in the first stage, the question of such an optimisation does not arise but, if it be prescribed in the last stage, the optimum staging for maximum burnout velocity is carried out by choosing equal payload ratios $(\pi)$ in all stages except in the last such that

$$
\varepsilon+(1-\varepsilon) \pi=\tau_{\text {in }} \pi^{n}
$$

similarly, for equal partial structural factors $(\varepsilon),(42 a)$ and $(42 b)$ lead to $\pi_{j}=\pi$, where $j=1,2,3 \ldots(r-1)$.

The maximum burnout velocity with a given final thrust-to-weight ratio in an arbitrary stage, the payload ratios of the last $(n-r)$ stages and equal structural factors in all stages, call for equal partial payload ratios $(\pi)$ in all the stages preceding that stage and a partial payload ratio in that stage such that

$$
\begin{aligned}
& \varepsilon+(1-\varepsilon) \pi=\tau_{f r} \pi^{r}\left\{\varepsilon+(1-\varepsilon) \pi_{r}\right\} \\
& \tau_{f r}\left\{\varepsilon+(1-\varepsilon) \pi_{r}\right\}=1-\varepsilon \sum_{k=r+1}^{n}\left[\left(1-\pi_{k}\right) \pi_{k-1} \pi_{k-2} \ldots \pi_{r}\right] / \pi_{r} .
\end{aligned}
$$

Equation (46b) reduces to the form, if the payload ratios of the last $(n-r)$ stages are equal $\left(\pi^{\prime}\right)$ :

$$
\tau_{f r}\left\{\varepsilon+(1-\varepsilon) \pi_{r}\right\}=1-\varepsilon\left(1-\pi^{\prime n-r}\right)
$$

Obviously, in this case, if the final thrust-to-weight ratio be given to the last ( $n$ th) stage,

$$
\begin{aligned}
& \varepsilon+(1-\varepsilon) \pi=\pi^{n}, \\
& \varepsilon+(1-\varepsilon) \pi_{n}=1 / \tau_{f r},
\end{aligned}
$$


i.e., such an optimum staging is due to equal partial payload ratios in all the stages preceding the last stage and a particular payload ratio in the last stage, given by (46d) and (46e). With the prescribed final thrust-to-weight ratio in the first stage, there exists a payload ratio $\left(\pi_{1}\right)$ in that stage, which maximizes the burnout velocity and is obtained from $(46 b) /(46 c)$ after the substitution $r=1$.

\section{Optimisation of partial payload ratios for maximum overall payload ratio with a given overall burnout velocity}

Case1: With a given initial thrust-to-weight ratio in the $r$ th stage the overall payload ratio for $n$ stages is given by

$$
\begin{aligned}
\pi_{0} & =\pi_{1} \pi_{2} \pi_{3} \ldots \pi_{n} \\
\text { or } \quad \log \pi_{0} & =\sum_{k=1}^{n} \log \pi_{k}
\end{aligned}
$$

Now, with a given burnout velocity $u$ the optimisation of $\pi_{0}$ leads to the optimisation of the function $U$ represented by

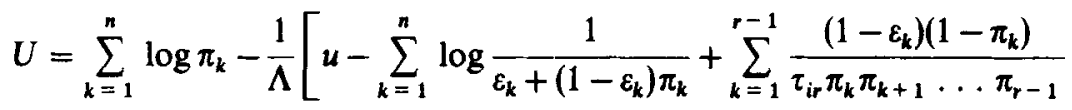

$$
\begin{aligned}
& \left.+\frac{\left(1-\varepsilon_{r}\right)\left(1-\pi_{r}\right)}{\tau_{i r}}+\sum_{k=r+1}^{n} \frac{\left(1-\varepsilon_{k}\right)\left(1-\pi_{k}\right) \pi_{k-1} \pi_{k}-2 \ldots \pi_{r}}{\tau_{i r}}\right]
\end{aligned}
$$

where $\Lambda$ is the Lagrangian multiplier.

Hence, the optimisation equations are obtained as

(i) For $j \leqslant r-1$,

$$
\begin{aligned}
& \frac{\partial U}{\partial \pi_{j}}=\frac{1}{\pi_{j}}+\frac{1}{\Lambda}\left[\frac{-\left(1-\varepsilon_{j}\right)}{\varepsilon_{j}+\left(1-\varepsilon_{j}\right) \pi_{j}}+\sum_{i=1}^{j-1} \frac{\left(1-\varepsilon_{i}\right)\left(1-\pi_{i}\right)}{\tau_{i r} \pi_{i} \pi_{i+1} \ldots \pi_{j}^{2} \ldots \pi_{r-1}}\right. \\
& \left.\quad+\frac{1-\varepsilon_{j}}{\tau_{i r} \pi_{j}^{2} \pi_{j+1} \ldots \pi_{r-1}}\right]=0 .
\end{aligned}
$$

(ii) For $j=r$,

$$
\begin{aligned}
\frac{\partial U}{\partial \pi_{r}}= & \frac{1}{\pi_{r}}+\frac{1}{\Lambda}\left[\frac{-\left(1-\varepsilon_{r}\right)}{\varepsilon_{r}+\left(1-\varepsilon_{r}\right) \pi_{r}}+\frac{1-\varepsilon_{r}}{\tau_{i r}}\right. \\
& \left.-\sum_{k=r+1}^{n} \frac{\left(1-\varepsilon_{k}\right)\left(1-\pi_{k}\right) \pi_{k-1} \pi_{k-2} \ldots \pi_{r}}{\tau_{i r} \pi_{r}}\right]=0 .
\end{aligned}
$$

(iii) For $r+1 \leqslant j \leqslant n-1$,

$$
\begin{aligned}
& \frac{\partial U}{\partial \pi_{j}}=\frac{1}{\pi_{j}}+\frac{1}{\Lambda}\left[\frac{-\left(1-\varepsilon_{j}\right)}{\varepsilon_{j}+\left(1-\varepsilon_{j}\right) \pi_{j}}\right. \\
& \left.-\sum_{i=j+1}^{n} \frac{\left(1-\varepsilon_{i}\right)\left(1-\pi_{i}\right) \pi_{i-1} \pi_{i-2} \ldots \pi_{r}}{\tau_{i r} \pi_{j}}+\frac{\left(1-\varepsilon_{j}\right) \pi_{j-1} \pi_{j-2} \ldots \pi_{r}}{\tau_{i r}}\right]=0 .
\end{aligned}
$$


(iv) For $j=n$,

$$
\frac{\partial U}{\partial \pi_{n}}=\frac{1}{\pi_{n}}+\frac{1}{\Lambda}\left[\frac{-\left(1-\varepsilon_{n}\right)}{\varepsilon_{n}+\left(1-\varepsilon_{n}\right) \pi_{n}}+\frac{\left(1-\varepsilon_{n}\right) \pi_{r} \pi_{r+1} \ldots \pi_{n-1}}{\tau_{i r}}\right]=0 .
$$

By means of the above $n$ optimisation equations and the constraint equation (14) in the nondimensional form, one can find the values of partial payload ratios and Lagrange's multiplier $\Lambda$ in terms of the prescribed initial thrust-to-weight ratio, partial structural factors and the given burnout velocity. Employing these values of partial payload ratios in (47), the corresponding maximum overall payload ratio can be evaluated. For equal structural factors $(\varepsilon)$ in all stages, $(49 a)$ reduces to the form:

$$
\frac{-\Lambda}{1-\varepsilon}=\frac{-\pi_{j}}{\varepsilon+(1-\varepsilon) \pi_{j}}+\frac{1}{\tau_{i r} \pi_{1} \pi_{2} \ldots \pi_{j} \ldots \pi_{r-1}},[j=1,2,3 \ldots(r-1)]
$$

The solution of these $(r-1)$ equations gives $\pi_{j}=\pi$

$$
\text { for all } j=1,2,3 \ldots(r-1) \text {. }
$$

Again, for $r+1 \leqslant j \leqslant n,(49 c)$ and (49d) lead to

$$
\frac{-\lambda}{1-\varepsilon}=\frac{-\pi_{j}}{\varepsilon+(1-\varepsilon) \pi_{j}}+\frac{\pi_{n} \pi_{n-1} \ldots \pi_{r+1} \pi_{r}}{\tau_{i r}}
$$

which implies $\pi_{j}=\pi^{\prime}$, for all $j=r+1, r+2, \ldots n$.

Now for $j=r$,

$$
\frac{-\lambda}{1-\varepsilon}=\frac{-\pi_{r}}{\varepsilon+(1-\varepsilon) \pi_{r}}+\frac{\pi_{n} \pi_{n-1} \ldots \pi_{r+1} \pi_{r}}{\tau_{i r}} .
$$

The relationships (52) to (54) can be combined to yield

$$
\pi_{r}=\pi^{\prime}
$$

Similarly because of (50) to (55), one can get

$$
\frac{\varepsilon\left(\pi^{\prime}-\pi\right)}{\left\{\varepsilon+(1-\varepsilon) \pi^{\prime}\right\}\{\varepsilon+(1-\varepsilon) \pi\}}+\frac{1}{\tau_{i r}}\left(\frac{1}{\pi^{\prime-1}}-\pi^{\prime n-r+1}\right)=0 .
$$

By the use of (51), (53) and (55) in (14), the latter reduces to

$$
\begin{aligned}
u= & (r-1) \log \frac{1}{\varepsilon+(1-\varepsilon) \pi}+(n-r+1) \log \frac{1}{\varepsilon+(1-\varepsilon) \pi^{1}} \\
& -\frac{1-\varepsilon}{\tau_{i r}}\left(\frac{1}{\pi^{r-1}}-\pi^{n-r+1}\right),
\end{aligned}
$$

where $1<r \leqslant n$.

With the given initial thrust-to-weight ratio in the arbitrary stage ( $r$ th), overall burnout velocity and equal structural factors in all stages, the maximum overall payload ratio is obtained if the partial payload ratio in each stage preceding that stage is the same and the partial payload ratio in each of the remaining $(n-r+1)$ is the same and these two kinds of optimum payload ratios are given by (56) and (57). The corresponding 
maximum overall payload ratio is given by

$$
\pi_{0}=(\pi)^{r-1}\left(\pi^{\prime}\right)^{n-r+1} .
$$

In this case, with the initial thrust-to-weight ratio prescribed in the first stage, the maximum payload ratio is due to its equal distribution among all the stages and each partial payload ratio satisfies (57) where $r=1$.

Case 2: With a given final thrust-to-weight ratio in the $r$ th stage the overall payload ratio for $n$ stages takes up the same form as in the previous case. Now, like the previous case the optimisation of $\pi_{0}$ [given by (47)] is the same as the optimisation of the function $U$ :

$$
\begin{aligned}
U= & \sum_{k=1}^{n} \log \pi_{k}-\frac{1}{\Lambda}\left[u-\sum_{k=1}^{n} \log \frac{1}{\varepsilon_{k}+\left(1-\varepsilon_{k}\right) \pi_{k}}\right. \\
& +\sum_{k=1}^{r-1} \frac{\left(1-\varepsilon_{k}\right)\left(1-\pi_{k}\right)}{\tau_{f r}\left\{\varepsilon_{k}+\left(1-\varepsilon_{k}\right) \pi_{k}\right\} \pi_{k} \pi_{k-1} \ldots \pi_{r-1}} \\
& \left.+\frac{\left(1-\varepsilon_{r}\right)\left(1-\pi_{r}\right)}{\tau_{f r}\left\{\varepsilon_{r}+\left(1-\varepsilon_{r}\right) \pi_{r}\right\}}+\sum_{k=r+1}^{n} \frac{\left(1-\varepsilon_{k}\right)\left(1-\pi_{k}\right) \pi_{k-1} \pi_{k-2} \ldots \pi_{r}}{\tau_{f r}\left\{\varepsilon_{r}+\left(1-\varepsilon_{r}\right) \pi_{r}\right\}}\right]
\end{aligned}
$$

where $\Lambda$ is the Lagrange multiplier.

The corresponding optimisation equations are given by

(i) For $j \leqslant r-1$,

$$
\begin{aligned}
\frac{\partial U}{\partial \pi_{j}}= & \frac{1}{\pi_{j}}+\frac{1}{\Lambda}\left[\frac{-\left(1-\varepsilon_{j}\right)}{\varepsilon_{j}+\left(1-\varepsilon_{j}\right) \pi_{j}}-\sum_{i=1}^{j-1} \frac{\left(1-\varepsilon_{i}\right)\left(1-\pi_{i}\right)}{\tau_{f r}\left\{\varepsilon_{r}+\left(1-\varepsilon_{r}\right) \pi_{r}\right\} \pi_{i} \pi_{i+1} \ldots \pi_{j}^{2} \ldots \pi_{r-1}}\right. \\
& \left.+\frac{\left(1-\varepsilon_{j}\right)}{\tau_{f r}\left\{\varepsilon_{r}+\left(1-\varepsilon_{r}\right)\right\} \pi_{j}^{2} \pi_{j+1} \ldots \pi_{r-1}}\right]=0
\end{aligned}
$$

(ii) For $j=r$,

$$
\begin{aligned}
\frac{\partial U}{\partial \pi_{r}}= & \frac{1}{\pi_{r}}+\frac{1}{\Lambda}\left[\frac{-\left(1-\varepsilon_{r}\right)}{\varepsilon_{r}+\left(1-\varepsilon_{r}\right) \pi_{r}}+\frac{1-\varepsilon_{r}}{\tau_{f r}\left\{\varepsilon_{r}+\left(1-\varepsilon_{r}\right) \pi_{r}\right\}^{2}}\right. \\
& \left.-\sum_{k=r+1}^{n} \frac{\varepsilon_{r}\left(1-\varepsilon_{k}\right)\left(1-\pi_{k}\right) \pi_{k-1} \pi_{k-2} \ldots \pi_{r}}{\tau_{f r}\left\{\varepsilon_{r}+\left(1-\varepsilon_{r}\right) \pi_{r}\right\}^{2} \pi_{r}}\right]=0 .
\end{aligned}
$$

(iii) For $r+1 \leqslant j \leqslant n-1$,

$$
\begin{aligned}
\frac{\partial U}{\partial \pi_{j}}= & \frac{1}{\pi_{j}}+\frac{1}{\Lambda}\left[\frac{-\left(1-\varepsilon_{j}\right)}{\varepsilon_{j}+\left(1-\varepsilon_{j}\right) \pi_{j}}-\sum_{i=j+1}^{n} \frac{\left(1-\varepsilon_{i}\right)\left(1-\pi_{i}\right) \pi_{i-1} \pi_{i-2} \ldots \pi_{r}}{\tau_{f r}\left\{\varepsilon_{r}+\left(1-\varepsilon_{r}\right) \pi_{r}\right\} \pi_{j}}\right. \\
& \left.+\frac{\left(1-\varepsilon_{j}\right) \pi_{j-1} \pi_{j-2} \ldots \pi_{r}}{\tau_{f r}\left\{\varepsilon_{r}+\left(1-\varepsilon_{r}\right) \pi_{r}\right\}}\right]=0 .
\end{aligned}
$$

(iv) For $j=n$,

$$
\frac{\partial U}{\partial \pi_{j}}=\frac{1}{\pi_{n}}+\frac{1}{\Lambda}\left[\frac{-\left(1-\varepsilon_{n}\right)}{\varepsilon_{n}+\left(1-\varepsilon_{n}\right) \pi_{n}}+\frac{\left(1-\varepsilon_{n}\right) \pi_{n} \pi_{n-1} \ldots \pi_{r}}{\tau_{f r}\left\{\varepsilon_{r}+\left(1-\varepsilon_{r}\right) \pi_{r}\right\} \pi_{n}}\right]=0 .
$$


In this case also, the $n$ optimum partial payload ratios can be determined from the above $n$ equations and the constraint equation (27) and hence the maximum payload ratio, by the use of (47). In case of the same partial structural factor $(\varepsilon)$ for all stages, in the same way as in the previous case, one gets

$$
\begin{aligned}
\pi_{j} & =\pi \text { for all } j=1,2,3, \ldots(r-1), \\
& =\pi^{\prime} \text { for all } j=r+1, r+2, \ldots n .
\end{aligned}
$$

Now for $j=r$, as a consequence of the conditions (61) and (62) substituted in (60a) to $(60 \mathrm{~d})$,

$$
\begin{aligned}
\frac{-\Lambda}{1-\varepsilon} & =\frac{-\pi_{r}}{\varepsilon+(1-\varepsilon) \pi_{r}}+\frac{\pi_{r}}{\tau_{f r}\left\{\varepsilon+(1-\varepsilon) \pi_{r}\right\}^{2}}-\frac{\varepsilon\left(1-\pi^{\prime n-r}\right) \pi_{r}}{\tau_{f r}\left\{\varepsilon+(1-\varepsilon) \pi_{r}\right\}^{2}} \\
& =\frac{-\pi}{\varepsilon+(1-\varepsilon) \pi}+\frac{1}{\tau_{f r}\left\{\varepsilon+(1-\varepsilon) \pi_{r}\right\} \pi^{r-1}} \\
& =\frac{-\pi^{\prime}}{\varepsilon+(1-\varepsilon) \pi^{\prime}}+\frac{\pi^{\prime \prime-\pi_{r}}}{\tau_{f r}\left\{\varepsilon+(1-\varepsilon) \pi_{r}\right\}}
\end{aligned}
$$

which can again be written as

and

$$
\frac{\varepsilon\left(\pi_{r}-\pi^{\prime}\right)}{\varepsilon+(1-\varepsilon) \pi^{\prime}}=\frac{\pi_{r}(1-\varepsilon)\left(1-\pi^{\prime n-r} \pi_{r}\right)}{\tau_{f r}\left\{\varepsilon+(1-\varepsilon) \pi_{r}\right\}}
$$

nd $\frac{\varepsilon\left(\pi-\pi^{\prime}\right)}{\{\varepsilon+(1-\varepsilon) \pi\}\left\{\varepsilon+(1-\varepsilon) \pi^{\prime}\right\}}=\frac{1}{\tau_{f r}\left\{\varepsilon+(1-\varepsilon) \pi_{r}\right\}}\left(\frac{1}{\pi^{r-1}}-\pi^{\prime n-r} \pi_{r}\right)$,

and (27) becomes

$$
\begin{aligned}
u= & (r-1) \log \frac{1}{\varepsilon+(1-\varepsilon) \pi}+\log \frac{1}{\varepsilon+(1-\varepsilon) \pi_{r}}+(n-r) \log \frac{1}{\varepsilon+(1-\varepsilon) \pi^{\prime}} \\
& -\frac{(1-\varepsilon)}{\tau_{f r}\left\{\varepsilon+(1-\varepsilon) \pi_{r}\right\}}\left(\frac{1}{\pi^{r-1}}-\pi_{r} \pi^{\prime n-r}\right)
\end{aligned}
$$

With the given final thrust-to-weight ratio in the $r$ th stage $(1<r<n)$, burnout velocity and equal structural factors in all stages, the maximum overall payload ratio is due to the optimum distribution of equal partial payload ratios $(\pi)$ among the first $(r-1)$ stages, a particular payload ratio $\pi_{r}$ in the $r$ th stage $(1<r<n)$ and equal payload ratios in the last $(n-r)$ stages. These three optimum payload ratios are obtained by solving (64), (65) and (66). If, the other conditions remaining the same, the final thrust-to-weight ratio be prescribed in the first stage, the optimum payload ratio associated with the first stage and that associated with each of the subsequent stages are obtained by putting $r=1$ in the equation involving $\pi_{r}$ and $\pi^{\prime}$ of (63) and also in (66), and the maximum overall payload ratio becomes

$$
\pi_{0}=\pi_{1} \pi^{\prime n-1}
$$

Similarly, with the given final thrust-to-weight ratio in the last stage, the corresponding two kinds of optimum payload ratios exist, the first kind in each stage preceding the last stage and the second kind in the last stage, and those that satisfy the equation 
involving $\pi_{r}$ and $\pi$ of (63) and (66) with $r=n$ such that the overall maximum payload ratio becomes

$$
\pi_{0}=\pi^{n-1} \pi_{n}
$$

Conversely, for the given initial/final thrust-to-weight ratio in the $r$ th stage, overall payload ratio and partial structural factors, there exist optimum partial payload ratios in all the stages which maximise the overall burnout velocity. However, from the engineering point of view the optimisation problem investigated in this section is more important than that in the preceding section.

\section{Conclusion}

The comparison of Miele's analysis with the present analysis reveals that with the same initial thrust-to-weight ratio, similar distribution of payload ratios, structural factors and equivalent exhaust velocities among the stages, the overall burn period with equal thrusts in all stages is less than that with equal initial/final thrust accelerations in all stages (thrusts decreasing in successive stages), as a consequence of which the present design leads to greater overall burnout velocity than that in Miele's design. It can also be noted that for the same equivalent exit velocities, payload ratios and structural factors in all the stages, the burn time of each stage in Miele's analysis is constant because of the same initial/final thrust-to-weight ratio in each stage while such partial burn time decreases in subsequent upper stages in the present analysis.

Finally, it is obvious that since the optimal staging under given conditions is due to a class of propellant mass distribution among the optimised stages in conformity with the mass of structural components to be discarded from each stage, constant thrust programming in a multistage vehicle also admits of optimisation criteria. This fact has also been established in the foregoing two sections of this paper.

\section{References}

Barrere M 1960 Rocket propulsion (Princeton: Elsevier) pp. 738-50

Miele A 1962 Flight mechanics (Reading Mass: Addison-Wesley) Vol. 1, pp. 358-79

Ruppe O H 1966 Introduction to astronautics (New York: Academic Press) Vol. I, pp. 26-50 